\title{
Left Ventricular Rupture Associated With Takotsubo-like Left Ventricular Dysfunction (Apical Ballooning)
}

\author{
Ryotaro Yamada, MD, Nozomi Watanabe, MD, Teruyoshi Kume, MD, \\ Takahiro Kawamoto, MD, Noriko Okahashi, MD, Nozomi Wada, MD, \\ Yuji Koyama, MD, Eiji Toyota, MD, Hiroyuki Okura, MD, \\ and Kiyoshi Yoshida, MD \\ Department of Cardiology, Kawasaki Medical School, Kurashiki, Japan
}

\begin{abstract}
A 71-year-old woman was admitted with sudden-onset shoulder and back pain. The electrocardiograph showed sinus rhythm with ST-segment elevation in leads $V_{46}$ and abnormal $\mathrm{Q}$ waves in leads $\mathrm{V}_{45 .}$. Echocardiography at the time of admission revealed akinesis in the left ventricular apical wall. However, coronary angiography did not reveal a stenotic lesion in any of the coronary arteries. Left ventriculography revealed apical ballooning with basal hyperkinesis. Ten hours after onset, the patient suddenly collapsed and went into cardiopulmonary arrest. This is a rare case of left ventricular rupture with takotsubo-like left ventricular dysfunction.

(J Echocardiogr 2006; 4: 59-62)
\end{abstract}

Key words: Takotsubo LV dysfunction, left ventricular rupture

A 71-year-old woman was admitted to our hospital with sudden-onset shoulder and back pain that had continued for 6 hours. Her past medical history included untreated hypertension for several years but no history of similar symptoms and no family history of cardiovascular disease. Her consciousness was clear; pulse rate was 94 beats/min; and blood pressure was 134/72 mmHg.

Auscultation of the heart and lungs were within normal limits. An electrocardiography (ECG) showed sinus rhythm with ST-segment elevation in leads $\mathrm{V}_{4-6}$ and abnormal $\mathrm{Q}$ waves in leads $\mathrm{V}_{45 .}$. Echocardiography at the time of admission revealed akinesis in the left ventricular (LV) apical wall and hyperkinesis in the basal wall with mitral valve systolic anterior wall motion. Laboratory data showed increased creatine

Received February 11, 2006; revision received April 11, 2006; accepted May 20, 2006

Address for correspondence: Ryotaro Yamada, MD

Department of Cardiology, Kawasaki Medical School,

577 Matsushima, Kurashiki 701-0192, Japan.

Telephone: +81-86-462-1111

Fax: +81-86-462-1199

E-mail: ryotaro@med.kawasaki-m.ac.jp

(C) 2006 Japanese Society of Echocardiography kinase concentration (CK=349 IU/L).

An acute myocardial infarction was suspected, and the patient underwent emergency cardiac catheterization. However, coronary angiography did not reveal any coronary artery stenosis. Left ventriculography revealed LV apical ballooning with basal hyperkinesis. LV ejection fraction was $33 \%$. A diagnosis was subsequently made of transient takotsubo-like LV dysfunction (apical ballooning). Eight hours after the onset of symptoms, the patient was carried into the intensive care unit, and an ECG was performed, showing prolonged ST-segment elevation. Two hours later she suddenly collapsed and went into cardiopulmonary arrest with electromechanical dissociation. We attempted resuscitation with percutaneous cardiopulmonary support. Echocardiography revealed an anechoic zone around the heart, suggesting pericardial effusion due to cardiac rupture. The patient died 6 hours post-cardiac rupture. In accordance with her family's wishes, an autopsy was not performed.

\section{Discussion}

Clinical features of takotsubo-like transient LV dysfunction (LV apical ballooning) have been reported as follows: (1) abnormal reversible wall motion that 

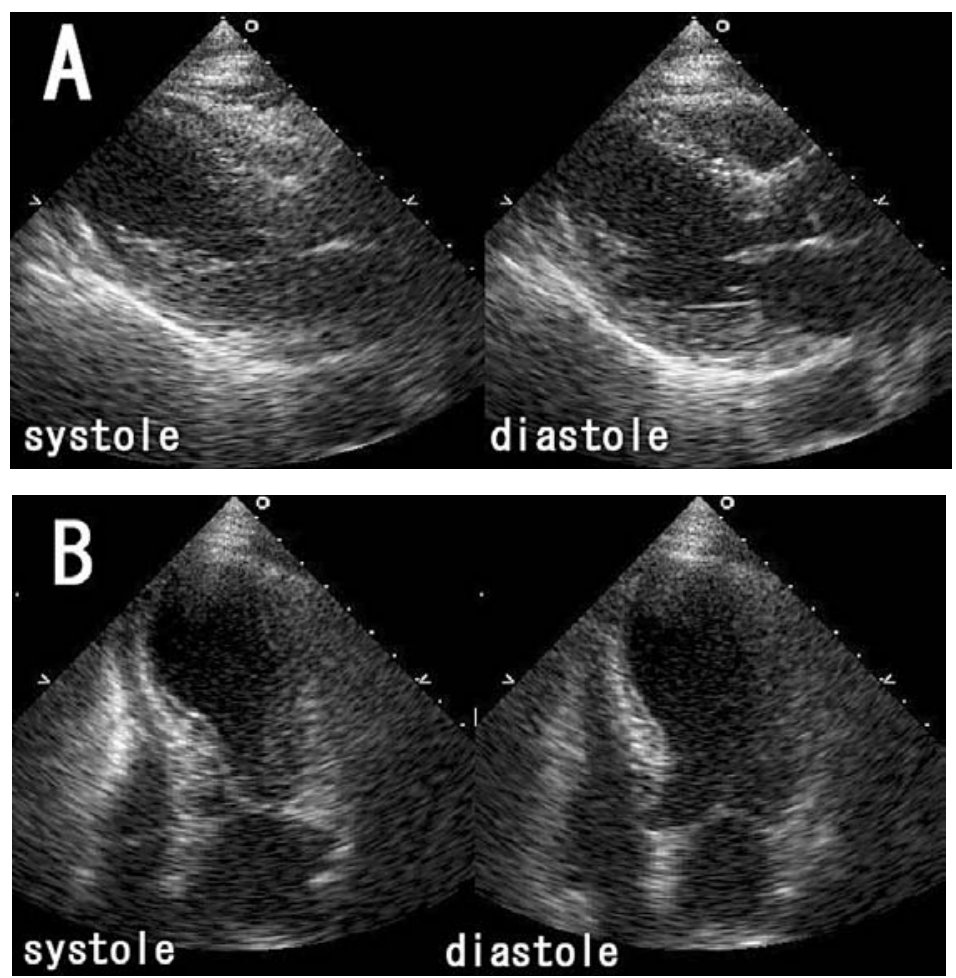

Fig. 1. Transthoracic echocardiography

(A, Parasternal long axis view; B, Four chamber view)

Echocardiography at the time of admission revealed akinesis in the left ventricular apical wall.
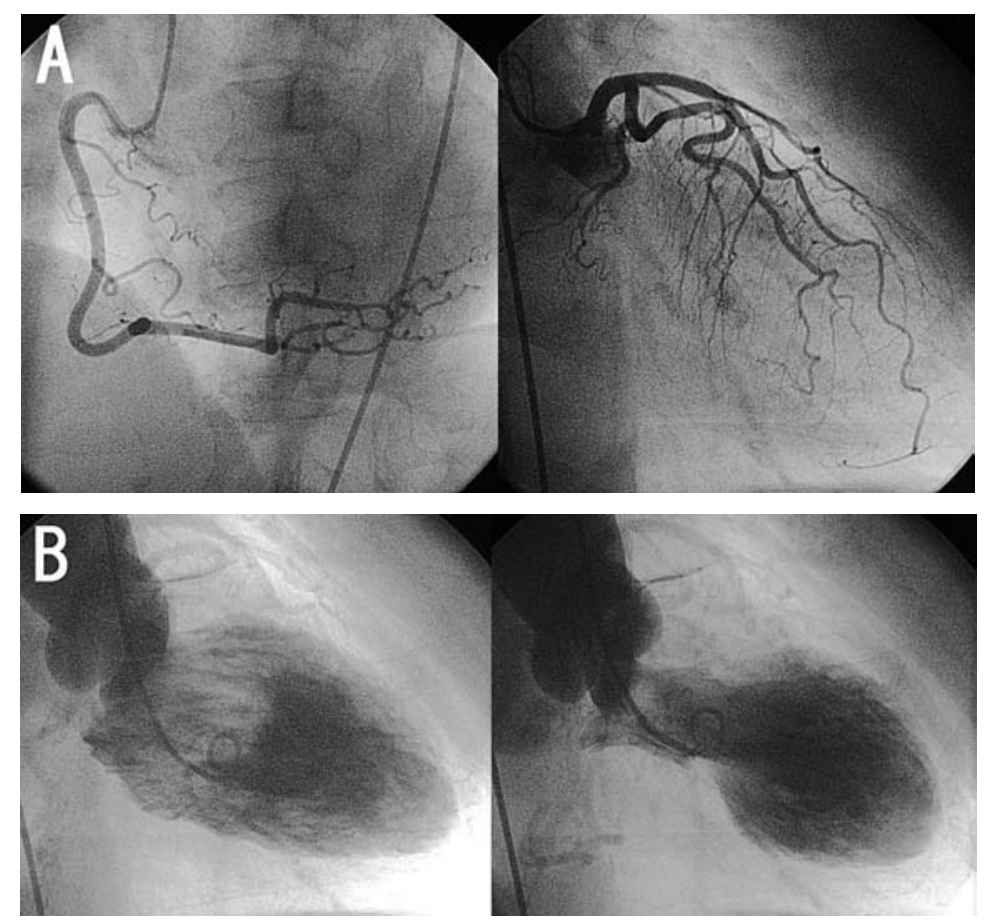

Fig. 2. Coronary angiography (A) and left ventriculography (B)

Coronary angiography did not reveal any coronary artery stenosis. Left ventriculography revealed apical ballooning with basal hyperkinesis. 
improves within a few weeks; (2) abnormal, transient ST-T segment on ECG; (3) minimal evidence of epicardial coronary artery stenosis, vasospasm, or microcirculatory disturbance; and (4) physical or emotional stress as trigger factors [1-4]. The reversibility of ventricular dysfunction in this disease is reminiscent of ischemic myocardial stunning, but LV apical balloon-

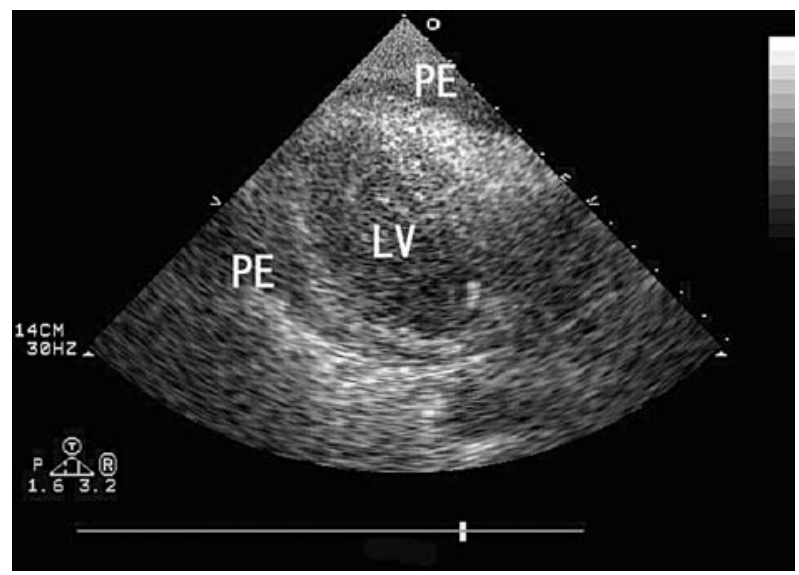

Fig. 3. Transthoracic echocardiography (LV, Left ventricle; PE, Pericardial effusion) Echocardiography revealed an anechoic zone around the heart, suggesting pericardial effusion due to cardiac rupture. ing is associated with patent coronary arteries even during the acute phase when ST-segment elevation is seen. The leads of ECG changes are not specific in this disease. In most cases, ST-segment elevation persists in the precordial leads for 12 hours or more, and pathologic $\mathrm{Q}$ waves and deep inverted $\mathrm{T}$ waves ultimately develop in the leads that initially showed STsegment elevation [4]. In our fatal case, we did not find any specific physical or emotional stress prior to the onset; reprolonged ST-segment elevation was observed for at least 10 hours in most precordial leads. We did not observe ECG or wall motion changes in the chronic stages of this patient because of her sudden death in the acute phase.

Several cases of LV rupture associated with LV apical ballooning have been previously reported $[1,2]$. In those cases, ST-segment elevation persisted in the precordial leads for several days or more. It has been reported that inflammatory infiltrates and myocyte necrosis were seen in the specimen from the rupture site in a previous case [2].

In the present case, abnormal $\mathrm{Q}$ wave and increased CK concentration were revealed, and these findings may be risk factors of cardiac rupture in LV apical ballooning. Furthermore, systolic anterior motion of the mitral valve could have resulted in the LV outflow
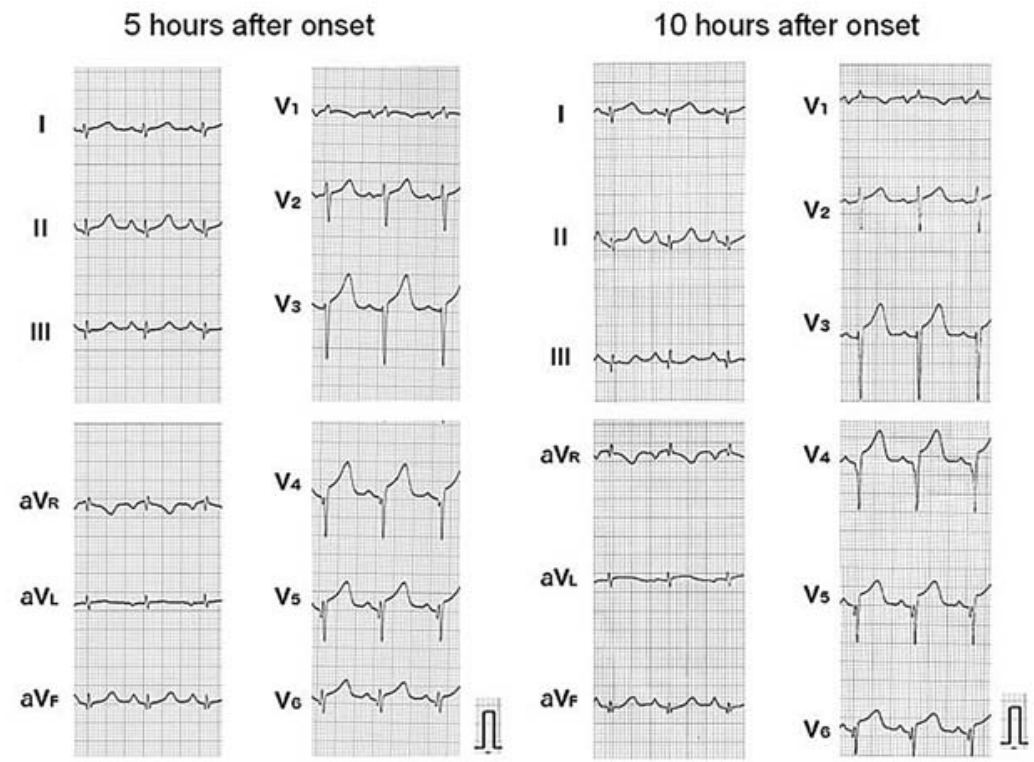

Fig. 4. Electrocardiograms (ECG) during the clinical course

Five hours after onset (at her family physician's office), ECG showed sinus rhythm with ST-segment elevation in leads $V_{46}$ and abnormal $Q$ waves in leads $V_{4.5}$. ECG still showed prolonged ST-segment elevation without T wave inversion when she was carried into the intensive care unit 10 hours after onset. 
obstruction with increased LV pressure, which may be a mechanism of cardiac rupture in this patient. Further investigation would be required to find out the mechanism and risk factors of cardiac rupture in LV apical ballooning, which is otherwise generally reversible and with good prognosis.

\section{References}

1. Yoshihiro J Akashi, Tamotsu Tejima, Harumizu Sakurada, et-al. Left Ventricular Rupture Associated With Takotsubo Cardiomyopathy. Mayo Clin Proc. 2004; 79: 821-824.

2. Yoshikazu Ohara, Yoshikazu Hiasa, Shinobu Hosokawa, et-al. Left Ventricular Free Wall Rupture in Transient Left Ventricular Apical Balloning. Circ J 2005; 69: 621-623.

3. Teruyoshi Kume, Takashi Akasaka, Takahiro Kawamoto, et-al. Relationship Between Coronary Flow Reserve and Recovery of Regional Left Ventricular Function in Patients With Tako-Tsubo-Like Transient Left Ventricular Dysfunction. Journal of Cardiology 2004; 43: 123-129.

4. Riyo Ogura, Yoshikazu Hiasa, Takefumi Takahashi, et-al. Specific Findings of the Standard 12-Lead ECG in Patients With ‘Takotsubo’ Cardiomyopathy Comparison With the Findings of Acute Anterior Myocardial Infarction. Circ J 2003; 67: 687-690. 